【研究简报】

\title{
湖泊沉积研究中一种定量估算 陆源有机碳的方法
}

\begin{abstract}
钱君龙 ${ }^{(1)}$ 茾民苏薛 滨 ${ }^{(1)}$ 陈如松 (2) 柯善哲(2) (1)中国科学院南京地理与湖泊研究所, 湖泊沉积与环境开放研究实验室, 南京 210008; (2)南京大学物理学系, 南京 210008) 关策词 湖泊沉积 陆潧有机碳 有机 $\mathbf{C} / \mathbf{N}$

湖泊沉积研究中常把总有机质含量 (TOM) 或总有机碳含量 (TOC) 作为判识湖泊环境的 一个重要指标. 事实上, 湖泊沉积物中的有机碳来源广泛, 简单地可分出内源和陆源两部分.
\end{abstract}

出, 1787 站岩心沉积物放射虫氧同位素变化特征与浮游有孔虫氧同位素标准曲线中 $0 \sim 27$ $\mathrm{Ma}$ 段具有良好的相似性.

由此,图 2(b)曲线显示出, $27 \mathrm{Ma}$ 以来 1787 站岩心氧同位素变化经历了 7 次冷暖交替变 化. 暧期对应的时间尺度分别为 $27 \sim 25,16 \sim 15,10 \sim 9,7.5 \sim 6.5,5.5 \sim 4.5,4 \sim 3$ 和 0.5 Ma. 这些时间尺度范围与文軒 $[5]$ 的暖期对应范围大致相当.

\section{4 结论}

(1) 本次以太平洋超过 $5000 \mathrm{~m}$ 水深的岩心沉积物为试样, 进行氧、硅稳定同位素测定和 研究, 是大洋中 $\mathrm{CCD}$ 以下缺乏钙质生物壳体而用放射虫壳体 $\mathrm{SiO}_{2}$ 进行测试的成功尝试. $\delta^{18} \mathrm{O}_{\mathrm{SMOW}}$ 分布在 $(21.2 \sim 34.5) \times 10^{-3}$ 之间, $\delta^{30} \mathrm{Si}_{\mathrm{NBS} 28}$ 在 $(0.2 \sim 1.2) \times 10^{-3}$ 之间变化, 与大洋 其他生物壳体的氧、硅同位素组成有一定的衔接和区别.

(2) $\delta^{18} \mathrm{O}-\delta^{30} \mathrm{Si}$ 值关系图, 清楚地反映了 1787 站岩心数据分布呈现两组, 基本以岩心沉积 层的 $160 \mathrm{~cm}$ 为分界. 两种同位素曲线也真实地记录了这一点, 反映了界线上下大洋环境和地 层层序上的演化差异.

(3) $\delta^{18} \mathrm{O}$ 值的对比研究认为, 1787 站岩心反映了 $27 \mathrm{Ma}$ 以来大洋经历了 7 次明显的气候 冷暧变化, 随之而来的全球冰体积和海平面也频繁地发生变化.

\section{参考文献}

1 Clayton R N. High temperature isotope effects in the early solar system. In: Reviews in Mineralogy, $1986.129 \sim 139$

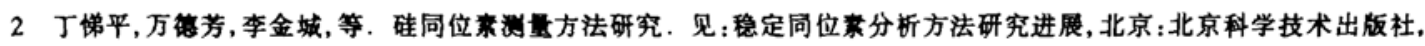
1992. $1 \sim 8$

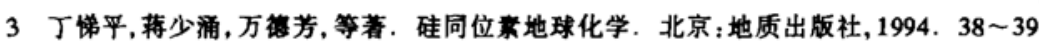

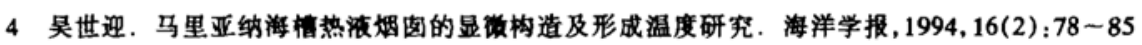

5 Prentice M L. Matthews R K. Cenozoic ice-volume history: development of a composite oxygen isotope record. Geology. 1988, 16:963 966 
内源有机碳主要是湖泊自身浮游生物的贡献, 陆源有机碳是由湖泊四周河流搬运入湖, 主要是 流域范围内陆生植物的贡献. 后者直接受控于流域气候与环境状况, 例如降水量大, 地表径流 发育, 植被茂盛, 则湖泊沉积有机质中陆源成分大. 因此, 只有陆源有机碳比重的大小才是气 候环境的更为有效的代用指标之一, 可以用来间接反映降水量丰沛的程度, 而陆源有机碳含量 是无法直接测定的, 如何定量估算沉积物总有机碳中陆源有机碳的含量已成为湖泊沉积研究 中一个重要的课题.

\section{1 方法}

不同来源的有机质, 其总有机碳氮比值 $(\mathrm{C} / \mathrm{N})$ 有显著差别. 已有研究表明, 陆生高等植物 的 $\mathrm{C} / \mathrm{N}$ 为 $14 \sim 23$, 甚至大于 30 , 而湖泊浮游生物的 $\mathrm{C} / \mathrm{N}$ 为 $6 \sim 7^{[1]}$. 基于这一研究, 考虑到研 究湖泊沉积柱状样中可以获得剖面沿程总有机碳 (TOCC) 和总有机氮 (TON) 的实测数据, 我 们考虑应用下面二元模型来定量估算两个来源的有机碳含量及其百分数.

设实测的钻孔 $i$ 层深度沉积物 TOC, TON 含量分别为 $\mathrm{C}(i), \mathrm{N}(i)$, 若令其中来自陆生和 内生的分别为 $\mathrm{C}_{1}(i), \mathrm{N}_{\mathrm{l}}(i) ; \mathrm{C}_{\mathrm{s}}(i), \mathrm{N}_{\mathrm{s}}(i)$, 则有

$$
\begin{aligned}
& \mathrm{C}(i)=\mathrm{C}_{\mathrm{l}}(i)+\mathrm{C}_{\mathrm{s}}(i), \mathrm{N}(i)=\mathrm{N}_{\mathrm{l}}(i)+\mathrm{N}_{\mathrm{s}}(i), \\
& \mathrm{C}_{\mathrm{l}}(i) / \mathrm{N}_{\mathrm{l}}(i)=R_{l}(i), \mathrm{C}_{\mathrm{s}}(i) / \mathrm{N}_{\mathrm{s}}(i)=\mathrm{R}_{\mathrm{s}}(i) .
\end{aligned}
$$

参数 $R_{1}(i)$ 和 $R_{\mathrm{s}}(i)$ 是深度为 $i$ 的沉积物中来自陆源和内源的碳氮比. 由 (1) 式可解得

$$
\begin{aligned}
& \mathrm{N}_{\mathrm{f}}(i)=\left[\mathrm{C}(i)-R_{\mathrm{s}}(i) \mathrm{N}(i)\right] /\left[R_{\mathrm{l}}(i)-R_{\mathrm{s}}(i)\right], \\
& \mathrm{N}_{\mathrm{s}}(i)=\left[\mathrm{C}(i)-R_{\mathrm{l}}(i) \mathrm{N}(i)\right] /\left[R_{\mathrm{s}}(i)-R_{\mathrm{l}}(i)\right], \\
& \mathrm{C}_{\mathrm{l}}(i)=R_{\mathrm{l}}(i)\left[\mathrm{C}(i)-R_{\mathrm{s}}(i) \mathrm{N}(i)\right] /\left[R_{\mathrm{l}}(i)-R_{\mathrm{s}}(i)\right], \\
& \mathrm{C}_{\mathrm{s}}(i)=R_{\mathrm{s}}(i)\left[\mathrm{C}(i)-R_{\mathrm{l}}(i) \mathrm{N}(i)\right] /\left[R_{\mathrm{s}}(i)-R_{\mathrm{l}}(i)\right] .
\end{aligned}
$$

因此参数 $R_{l}(i)$ 和 $R_{s}(i)$ 的取值直接影响 (2) 式的结果. 上面提及的报道结果为平均值, 零级近似可取 $R_{1}(i)=R_{1}=23$ 和 $R_{\mathrm{s}}(i)=R_{\mathrm{s}}=6$, 但这组参数值, 可能导致 (2) 式的计算结果 出现负值, 考虑到 $\mathrm{C}_{\mathrm{f}}(i), \mathrm{N}_{\mathrm{l}}(i) ; \mathrm{C}_{\mathrm{s}}(i), \mathrm{N}_{\mathrm{s}}(i)$ 均应非负的, 故需要调整 $R_{1}$ 和 $R_{\mathrm{s}}$ 的取值. 根 据文献报道值, $R_{1}>R_{s}$, 故要求

$$
R_{\mathrm{s}} \leqslant \mathrm{C}(i) / \mathrm{N}(i), \quad R_{\mathrm{l}} \geqslant \mathrm{C}(i) / \mathrm{N}(i) .
$$

可取零级近似值

$$
\begin{aligned}
& R_{1}=\max [\mathrm{C}(i) / \mathrm{N}(i)]+\Delta_{l}, \\
& R_{\mathrm{s}}=\min [\mathrm{C}(i) / \mathrm{N}(i)]-\Delta_{\mathrm{s}},
\end{aligned}
$$

其中 $\Delta_{1}$ 和 $\Delta_{\mathrm{s}}$ 是调节参数, 可由 (2)式的计算结果和其它有关结果作比较来确定.

\section{2 实例分析}

运用上述二元模型, 对青藏高原东北部若尔盖盆地 RM 孔进行了陆源有机碳的分析研 究. RM 孔位于盆地沉积中心, 孔位 $33^{\circ} 57^{\prime} \mathrm{N}, 102^{\circ} 21^{\prime} \mathrm{E}$, 地面高程 $3410 \mathrm{~m}$ a.s. 1 . , 孔深 310.46 m. TOC 的测试采用重铬酸钾容量法 (外加热法) ${ }^{[2]}$; 沉积物中的无机氮含量很低, 故用沉积 物总氮含量近似代替 TON, 总氮的测试采用过硫酸盐-紫外分光光度法 ${ }^{[3]}$.

取 RM 孔深度 $i$ 介于 $4.3 \sim 31.71 \mathrm{~m}$, 共 115 个样品的 $\mathrm{C}(i), \mathrm{N}(i)$, 其 $\mathrm{C}(i) / \mathrm{N}(i)$ 的最大 值和最小值分别为 22.264 和 3.569 , 取 $R_{1}=22.264, R_{\mathrm{s}}=3.569$ 及 $R_{1}=23.0, R=3.0$, 根据 
(2) 式得出结果分别为 $T_{O C}(i)$, $\operatorname{TOC}_{1}^{\prime}(i)$ (见图 1). 对比分析可见, 两者 结果比较接近, 数据较稳定, 来自陆源的 碳百分比由两组系数计算结果误差大都 在 $4 \%$ 以内.

为了验证该方法的有效性, 我们进 行了 $\operatorname{TOC}(i) / \mathrm{TN}(i), \operatorname{TOC}_{1}(i) /$ $\mathrm{TOC}(i)$ 与 $\mathrm{CD} / \mathrm{TC}$ 比值的比较 (图 2). $\mathrm{CD} / \mathrm{TC}$ 也即沉积物中叶绿素及其衍生 物总量与类胡萝卜素总量的比值, $\mathrm{CD} / \mathrm{TC}$ 是衡量异地与内生有机质平衡 的有效指标之一. $\mathrm{CD} / \mathrm{TC}$ 值高, 表明有 丰富的陆源有机质, 反之, 陆源有机质含 量低 ${ }^{[4]}$.

从图 1 可见, $\operatorname{TOC}(i)$ 和 $\operatorname{TOC}_{1}(i)$ 的 变化曲线有非常好的正相关关系, 说明 模式计算出的陆源有机碳含量 $\operatorname{TOC}_{1}(i)$ 和沉积物中有机碳总量 $\operatorname{TOC}(i)$ 一样, 均

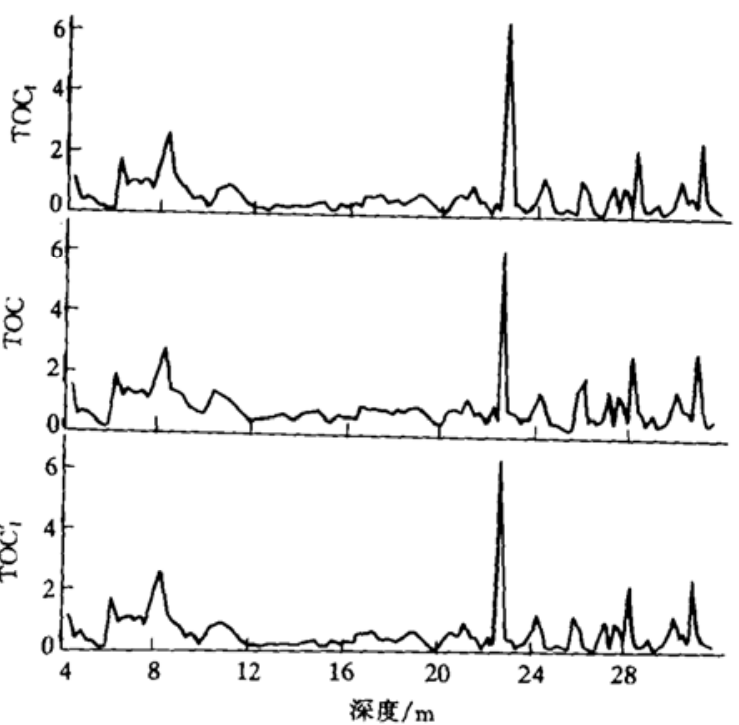

图 $1 \mathrm{RM}$ 孔沉积物 $\operatorname{TOC}(i), \operatorname{TOC}_{1}(i)$, $\operatorname{TOC}_{1}{ }^{\prime}(i)$ 值的分布 可作为判析湖泊环境的一种指标, 同时也反映了若尔盖古湖长期处于基本稳定的湖泊环境, 保 存条件变化不大 ${ }^{[s]}$.

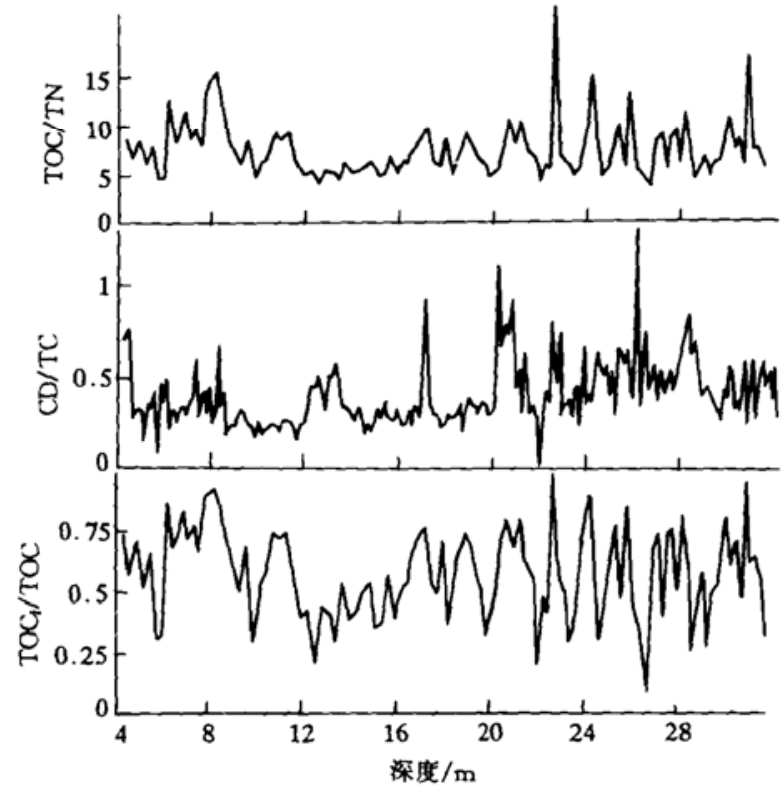

图 2 RM 孔沉积物 $\operatorname{TOC}(i) / \operatorname{TN}(i), \operatorname{TOC}(i) / \operatorname{TOC}(i)$ 与 $\mathrm{CD} / \mathrm{TC}$ 比值的分布

从图 2 可见, $\operatorname{TOC}_{1}(i) / \operatorname{TOC}(i)$ 与 $\mathrm{CD} / \mathrm{TC}$ 比值也有较好的正相关关系, 从 另一个侧面证明了该模式的可行性.

\section{3 结论}

湖泊沉积埴藏了湖泊自身以及区域 范围的古气候与古环境演化的信息, 这 也相应地导致古湖泊研究中各项代用指 标的复杂性与多解性. 如何纯化这些指 标的古气候古环境指示意义, 也成为湖 泊沉积领域一个亟待解决的问题. 过去 较多的指标研究也能定性地区分湖泊沉 积物中陆源与内源有机质组分的多塞, 如有机质 $\delta^{13} \mathrm{C}, \mathrm{CD} / \mathrm{TC}$, 本文采用的二 元模型成功地找到了一种定量估算陆源 有机碳含量的方法, 但如何确定调节参 数 $\Delta_{1}$ 和 $\Delta_{\mathrm{s}}$, 还需要今后进一步工作. 
【研究简报】

\title{
与低 ${ }^{18} \mathrm{O}$ 岩浆有关的矽卡岩型铅锌矿床
}

\author{
牟保磊郡济安王关玉阎国翰艾永富 \\ (北京大学地质系, 北京 100871)
}

\section{关铜诋 ${ }^{18} \mathrm{O}$ 岩浆 矽卡岩 交代流体}

内蒙赤峰市以北约 $360 \mathrm{~km}$ 巴林左旗境内, 大兴安岭山脉的白音诺矽卡岩型铅锌矿床中, 矽卡岩矿物及与矽卡岩有关的火成岩极端贫 ${ }^{18} \mathrm{O}$. 该地区其他同类铅锌矿床也有这种现象. 这很值得重视研究.

由花岗闪长岩、石英角闪二长斑岩、二长花岗岩及正长斑岩组成外环, 以喷溢相流纹质晶 屑熔结凝灰岩组成内环及核部的白音诺侵入-火山岩杂岩体, 面积约 $110 \mathrm{~km}^{2}$. 侵入岩之间为 过渡关系. 火山岩由化学成分看从钾长流纹岩至英安岩, 以前者为主. 侵入岩及火山岩的 $\mathrm{Rb}-\mathrm{Sr}$ 等时线年龄各为 171 和 $160 \mathrm{Ma}^{1}{ }^{1}$. 在杂岩体的东北部, 侵人岩以岩枝、岩舌, 塎岩以岩舌 侵入或突入下二叠统黄岗梁组的凝灰粉砂质板岩、厚层大理岩、结晶灰岩, 在接触带上形成角 岩及矽卡岩. 矽卡岩中赋存大型-超大型铅锌矿床.

由花岗闪长岩至大理岩其交代剖面是: 长石化花岗闪长岩、长石绿䆑石矽卡岩、辉石石榴 石矽卡岩、石榴石辉石矽卡岩、辉石矽卡岩; 从塎岩至大理岩的交代剖面是: 长石化熔岩、辉石 石榴石矽卡岩、石榴石辉石硅灰石矽卡岩. 矽卡岩矿物中量最多的是辉石、石榴石和硅灰石. 辉石为透辉石一钻铁辉石一镭铝辉石系列. 石榴石以䥻铁榴石、钙铝榴石为主, 锤铝榴石在东矿 段丰富. 从时间上看矽卡岩形成之后又叠加由石英、长石、方解石、帘石、角闪石、符山石和少 量黑柱石等矿物组成的含水矽卡岩. 第三阶段为石英-硫化物主要成矿期. 硫化物以闪锌矿 为主, 其次是方铅矿、黄铜矿、黄铁矿等. 与熔岩有关的矽卡岩中还有锡石、磁铁矿.

根据矿物相平衡计算和包襄体均一法确定早期矽卡岩形成温度为 $650 \sim 500 \mathrm{C}$, 晚期矽卡 岩为 $480 \sim 350^{\circ} \mathrm{C}$, 石英-硫化物阶段为 $400 \sim 220^{\circ} \mathrm{C}$.

致谢纪圣谋同志在建模中给予帮助, 潘红等同志提供色素分析数据, 在此一并表示感谢. 本 工作为国家“登”计划 $(\mathrm{KJ} 85 \cdot 07 \cdot 02)$ 资助项目.

\section{考文献}

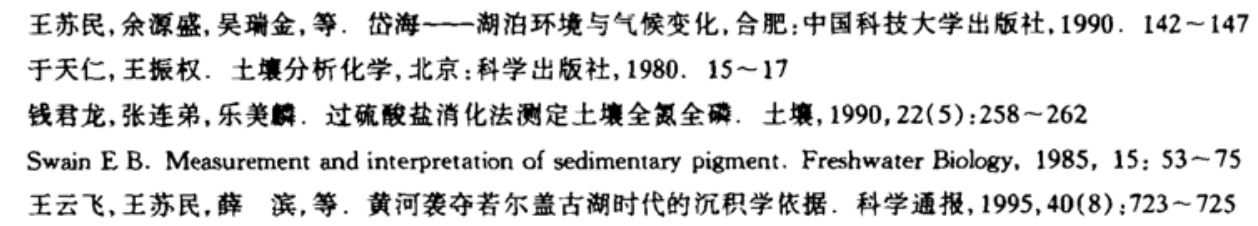

(1996-07-24 收稿, 1997-01-21 收修改稿)

1) 罗太阳、艾永富, 牟保否, 等, 内蒙古自治区巴林左䃆白音诺铅锌矿床成矿规律研究报告. 1991, 19 20 